\section{Sepsis Induced Pediatric Acute Respiratory Distress Syndrome (PARDS) - Are Biomarkers the Answer in a Resource Limited Setting?}

Sepsis is a life-threatening organ dysfunction due to a dysregulated host response to infection that can have myriad etiologies. Amongst the organ dysfunction caused, sepsisinduced acute respiratory distress syndrome (ARDS) is a common entity that has been associated with poor patient outcomes [1]. The pathogenesis of sepsis-induced ARDS is essentially a pulmonary manifestation of systemic inflammatory response syndrome (SIRS). More than $4 \%$ of all hospitalized patients less than 18 years and an approximate $8 \%$ of patients admitted to pediatric intensive care units (PICUs) in high- income countries have sepsis [2]. Amongst the organ dysfunctions mentioned, Pediatric acute respiratory distress syndrome (PARDS) accounts for an average mortality rate of $20-30 \%$ worldwide [3], and $70 \%$ in developing countries [4]. Gupta, et al. [5] reported sepsis as the precipitating cause in $37 \%$ of their patients with PARDS. Another study on outcomes and predictors of mortality in ARDS [6] highlighted the importance of inflammatory biomarkers at admission to predict patient outcomes.

Owing to significant data extrapolation from adult studies in sepsis and ARDS, there is a need to have dedicated research in this specific area. It is imperative to find a therapeutic answer that may predict the onset of PARDS, thereby alerting the intensivist, and subsequently shall lead to reduced morbidity and better patient outcomes. It therefore becomes quintessential to distinguish between pulmonary and nonpulmonary infection to better understand the epidemiology of sepsis and PARDS.

The enzyme-linked immunosorbent assay (ELISA) is the gold standard for biomarker measurement of plasma proteins [7]. Owing to the heterogeneity of PARDS, literature suggests combination of clinical indices and plasma biomarkers, that include IL-6, IL-8, soluble tumor necrosis factor receptor-1 (sTNFr-1), plasminogen activator inhibitor (PAI-1), angio- poeitin-2 (Ang-2), Soluble receptor for advanced glycation end products (sRAGE) at 6 and 24 hours of diagnosis [7]. Pediatric Acute Lung Injury Consensus Conference (PALICC) guidelines from 2015 [5] advocate serial monitoring of biomarkers to predict patient outcomes, thereby bringing bench to bedside.

PARDS is under-recognized due to lack of a universally accepted definition till now and this has led to under-and overestimation of its true prevalence. In resource-limited settings, an insight into markers-specific approach towards at risk patient population using combinations of plasma and clinical biomarkers in sepsis induced PARDS is likely to be the cornerstone of precision medicine, and eventually be the answer to the uncertainty that exists today.

SIDRA ISHAQUE
Pediatric Critical Care Medicine
Department of Pediatrics and Child Health
The Aga Khan University Hospital, Karachi, Pakistan.
sidra.ishaque@aku.edu

\section{REFERENCES}

1. Yehya N, Thomas NJ. Sepsis and pediatric acute respiratory distress syndrome. J Pediatr Intensive Care. 2019;8:32-41.

2. Ruth A, McCracken CE, Fortenberry JD, et al. Pediatric severe sepsis: Current trends and outcomes from the Pediatric Health Information Systems database. Pediatr Crit Care Med. 2014;15:828-38.

3. Khemani RG, Belani S, Leung D, et al. Pulse oximetry vs. $\mathrm{PaO} 2$ metrics in mechanically ventilated children: Berlin definition of ARDS and mortality risk. Intensive Care Med. 2015;41:94-102.

4. Schouten LR, Veltkamp F, Bos AP, et al. Incidence and mortality of acute respiratory distress syndrome in children: A systematic review and meta-analysis. Crit Care Med. 2016;44:819-29.

5. Gupta S, Sankar J, Lodha R, Kabra SK. Comparison of prevalence and outcomes of pediatric acute respiratory distress syndrome using pediatric acute lung injury consensus conference criteria and berlin definition. Front Pediatr. 2018;6:93.

6. Sharma SK, Gupta A, Biswas A, et al. Etiology, outcomes and predictors of mortality in acute respiratory distress syndrome from a tertiary care centre in north India. Indian J Med Res. 2016;143:782-92.

7. Carlton EF, Flori HR. Biomarkers in pediatric acute respiratory distress syndrome. Ann Transl Med. 2019;7:505. 